Oral presentation

Open Access

\title{
New mouse models for the analysis of cGMP signalling
} Robert Feil ${ }^{* 1}$, Susanne Feil ${ }^{1}$, Paul Franken ${ }^{2}$, Yann Emmenegger ${ }^{2}$, Mehdi Tafti ${ }^{2}$, Karin Weindl ${ }^{3}$, Sabine M Hölter ${ }^{3}$, Wolfgang Wurst ${ }^{3}$, Sonja Langmesser ${ }^{4}$, Urs Albrecht ${ }^{4}$, Michael Föller ${ }^{5}$, Florian Lang ${ }^{5}$, Silke Weber ${ }^{6}$ and Franz Hofmann ${ }^{6}$

Address: ${ }^{1}$ Interfakultäres Institut für Biochemie, Universität Tübingen, Germany, ${ }^{2}$ Center for Integrative Genomics, University of Lausanne, Switzerland, ${ }^{3}$ Institute of Developmental Genetics, GSF, Neuherberg, Germany, ${ }^{4}$ Institute of Biochemistry, University of Fribourg, Switzerland, ${ }^{5}$ Department of Physiology, Universität Tübingen, Germany and ${ }^{6}$ Institut für Pharmakologie und Toxikologie, TU München, Germany

Email: Robert Feil* - robert.feil@uni-tuebingen.de

* Corresponding author

from $3^{\text {rd }}$ International Conference on cGMP Generators, Effectors and Therapeutic Implications Dresden, Germany. 15-17 June 2007

Published: 25 July 2007

BMC Pharmacology 2007, 7(SuppI I):SI9 doi:I0.II86/I47I-22I0-7-SI-SI 9

This abstract is available from: http://www.biomedcentral.com/I47I-22I0/7/SI/SI9

(c) 2007 Feil et al; licensee BioMed Central Ltd.

The second messenger cGMP mediates beneficial and detrimental effects of nitric oxide and natriuretic peptides. Studies with conventional knockout mice showed that the cGMP-dependent protein kinase type I (cGKI) transduces many acute effects of cGMP, such as smooth muscle relaxation and inhibition of platelet aggregation. However, the short life expectancy of cGKI null mice precludes the analysis of long-term processes in adult mice. Recently, we have generated conditional tissue-specific cGKI knockout and rescue mice that can be studied throughout adulthood. These mouse models have uncovered previously unknown functions of cGMP/cGKI signalling in complex behavioural processes, such as object recognition, social discrimination and the regulation of sleep-wake activity. Moreover, a novel role of cGKI in erythrocyte death will be discussed. 